\title{
Adrenal Kortikal Karsinom’da Bilgisayarlı Tomografi Görüntüleme Bulguları
}

\section{Computed Tomography Findings in Adrenocortical Carcinoma}

iD

Emre Altınmakas ${ }^{1,2}$

${ }^{1}$ Koç Üniversitesi Tıp Fakültesi Radyoloji Anabilim Dalı, İstanbul, Türkiye

${ }^{2}$ Icahn School of Medicine at Mount Sinai, Department of Radiology, New York, USA

Öz

Amaç: Adrenal kortikal karsinomun (AKK) Bilgisayarlı Tomografi (BT) görüntüleme özelliklerini araștırmak.

Gereç ve yöntem: Patolojik olarak AKK tanısı almış 15 hastanın (K/E: 8/7, ortanca [aralık]: 53 yıl [31-74 yıl]) kalitatif ve kantitatif BT bulguları retrospektif olarak değerlendirildi. Lezyonların fonksiyonel durumlarına klinik notlardan ulaşıldı.

Bulgular: Hastaların patolojik tanısı cerrahi ( $\mathrm{n}=12)$ veya cerrahi eşliğinde biyopsi $(\mathrm{n}=3)$ ile konulmuştu. 9 lezyon (\%60) fonksiyonel, 6 lezyon (\%40) nonfonksiyoneldi. Fonksiyonel lezyonların 5'i androjen, 4'ü kortizon üretmekteydi. Kalitatif değerlendirmede 13 lezyon (\%93) nekroz, 3 lezyon (\%20) kalsifikasyon ve 1 lezyon (\%7) makroskobik yağ içeriyordu. Lezyonların tamamı iyi sınırlı olup heterojen kontrastlanmaktaydı. Lezyonların ortanca (aralık) boyutu 9,7 cm (6,3-18 cm) idi. Prekontrast, arteriyel faz, portal faz ve geç faz ortanca (aralık) dansite değerleri sırasıyla şöyleydi: 34 HU (22-41 HU), 46 HU (27-65 HU), 60 HU (29-90 HU), 48 HU (28-64 HU). 9 hastada 15. dakika geç faz elde olunmuştu. Ortanca (aralık) mutlak (MKYY) ve bağıl (BKYY) kontrast yıkanma yüzdeleri sırasıyla şöyleydi: \%48,8 (\%-3,8-62,5) ve \%21 (\%-1,5-30,8). Sadece bir lezyonun MKYY değeri \%60’in üzerindeydi.

Sonuç: AKK'lar BT'de genellikle büyük $(>6 \mathrm{~cm})$, iyi sınırlı, heterojen kontrastlanan, nekrotik kitleler şeklinde görülür. Makroskobik yağ ve kalsifikasyon içerebilir ve geç faz imajlarda sınırlı kontrast yıkanması gösterirler.

Anahtar kelimeler: adrenal kortikal karsinom,bilgisayarli tomografi,kontrast yıkanma yüzdesi

Abstract

Objective: To evaluate computed tomography (CT) imaging manifestations of adrenocortical carcinomas (ACC).

Materials and Methods: Qualitative, and quantitative CT findings of 15 patients (Eight women, seven men; median age [range], 53 years [31-74 years]) with 15 pathologically proven ACCs were retrospectively analyzed. Lesions' functional status were captured through clinical notes.

Results: Pathologic diagnosis was based on either surgery $(n=12)$ or surgical biopsy $(n=3)$. Nine lesions were functioning $(60 \%$, androgen [ $n=5]$ or cortisol $[\mathrm{n}=4]$ ) and 6 lesions were non-functioning (40\%). In qualitative CT analysis, $13(93 \%), 3(20 \%)$, and $1(7 \%)$ lesion had necrosis, calcification and macroscopic fat, respectively. All lesions showed well-defined borders and heterogeneous enhancement. Median [range] density on precontrast, arterial phase, portal phase, and 15-minute delay phase were $34 \mathrm{HU}(22-41 \mathrm{HU}), 46 \mathrm{HU}$ (27-65 HU), $60 \mathrm{HU}$ (29-90 HU), and $48 \mathrm{HU}$ (28-64 HU), respectively. Nine patients had 15-minute delay phase available. Median (range) absolute and relative percentage of enhancement wash-out (APEW and RPEW) values were 48.8\% (-3.8-62.5\%) and 21\% (-1.5-30.8\%). Only one lesion had an APEW above \%60.

Conclusion: ACCs usually manifest as, large ( $>6 \mathrm{~cm})$, well-defined, heterogeneously enhancing, necrotic masses on CT. These lesions may include calcification or macroscopic fat and tend to show limited wash-out on delay phase CT.

Keywords: adrenocortical carcinoma,computed tomography,percentage of enhancement wash-out

Yazışma Adresi: Emre Altınmakas, Topkapı, Koç Üniversitesi Hastanesi, Davutpaşa Cd. No:4, 34010 Zeytinburnu/İstanbul, Türkiye

E-Posta: emrealtinmakas@gmail.com

Alınma tarihi: 29.07.2021 / Kabul tarihi: 26.08.2021 / Yayımlanma tarihi: 20.09.2021

Adrenal Kortikal Karsinom'da BT Bulguları - Altınmakas

Genel Tip Derg 2021;31(3)299-303 


\section{Giriş}

Adrenal kortikal karsinomlar (AKK) adrenal bezin korteksinden kaynaklanan, nadir görülen, agresif tümörlerdir. Tüm kanser olgularının \%0,05-0,2'sini oluşturdukları bildirilmiştir (1, 2). Genellikle ya çocuk yaş grubunda ( $<5$ yaş) ya da 4.-5. dekadda ortaya çıkarlar $(3,4)$. Kadınlarda daha sık görüldükleri bildirilmiştir (2). Vakaların \%60'ını fonksiyonel tümörler oluşturmaktadır (3).

Günümüzde AKK'lerin tanısında sıklıkla Bilgisayarlı Tomografi (BT), Pozitron Emisyon Tomografisi-Bilgisayarlı Tomografi (PETBT) ve Manyetik Rezonans Görüntüleme (MRG) kullanılmaktadır. AKK'ler ilk tanı anında genellikle büyük olup ( $>6 \mathrm{~cm})$ nekroz, kanama, kalsifikasyon ve nadiren de makroskobik yağ içerebilirler (5). Bu açıdan adrenal lenfoma, feokromasitoma gibi adrenal tümörler ile adrenal bezi tutabilen tüberküloz ve mantar enfeksiyonları ile benzer özellik gösterirler (6-8). Bazen büyük ( $>6 \mathrm{~cm}$ ) adenomlar ve metastazlar AKK'ler ile ayırıcı tanıya girebilirler.

Adrenal lezyonların BT ile tanısında yukarıda bahsedilen kalitatif bulguların yanında bazı kantitatif bulgular da kullanılır. En sık kullananlardan birisi geç faz BT'deki kontrast yıkanma yüzdeleridir (KYY) (9). Adrenal bezin en sik görülen lezyonu olan adenomların adenom-dışı lezyonlardan daha fazla kontrast yıkanması gösterdiği bilinmektedir (9). Ancak bahsi geçen çalışmada adenom-dışı lezyonlar ağırlıklı olarak metastazlardan oluşmuş olup AKK'ler dahil edilmemiştir. Ayrıca KYY kriterlerinin AKK tanısındaki etkinliği üzerine çok az çalışma mevcut olup bu çalışmaların tamamında geç faz olarak genel kabul görmüş 15 . dakika yerine $\leq 10$. dakika görüntüler kullanılmıştır $(10,11)$.

Bizim çalışmamızda, patolojik tanı almış 15 AKK vakasının retrospektif BT görüntüleri değerlendirilerek, nadir görülen bu tümörün kalitatif ve kantitatif BT bulguları analiz edildi. İlave olarak, bu lezyonların daha önce literatürde bildirilmemiş olan 15. dakika kontrast yıkanma yüzdeleri raporland1.

\section{Gereç ve Yöntem}

\section{Hastalar}

Etik kurul onamı alınmasını takiben patoloji veri tabanında kayıtlı adrenal kortikal karsinom vakaları retrospektif olarak taranmış ve patolojik tanı öncesi 6 ay içerisinde BT görüntülemesi olan $>18$ yaş hastalar çalışmaya dahil edilmiştir. Yaş, cinsiyet ve hormonal fonksiyonel durum bilgisi kaydedilmiştir. Patolojik tanı 12 hastada cerrahi ile 3 hastada cerrahi eşliğinde biyopsi ile konulmuştu.

\section{BT tekniği ve değerlendirme}

BT incelemeler General Electric Light Speed 16 cihazı ile gerçekleştirilmişti. BT parametreleri şöyleydi: $300 \mathrm{~mA}, 120 \mathrm{kVp}$, kesit kalınlığ $2,5 \mathrm{~mm}$. Intravenöz yoldan $100-150 \mathrm{~mL}$ non-ionik kontrast maddenin (iohexol $300 \mathrm{mgI} / \mathrm{mL}$, GE Healthcare Inc., Princeton, NJ) 2,0-3,0 mL/ saniye hızda verilmesinden önce ve 30-40 saniye (arteriyel faz), 6070 saniye (portal faz) ve 15 dakika sonra (geç faz) görüntüler elde olundu. Abdomen radyolojisi alanında 10 yıldan fazla deneyimi olan bir radyolog tarafindan [EA] BT görüntüleri PACS sisteminde (Intelli Space PACS Radiology, v4.4, Philips, Canada [CA]) kalitatif ve kantitatif olarak değerlendirildi. Aksiyel düzlemde lezyonların uzun aksları ve uzun aksa dik olacak şekilde kısa aksları ölçüldü. Eski 20
HU'nun altında dansite değerine sahip alanların yağ olduğu kabul edildi. Kontrast sonrası fazlardaki dansite değeri prekontrast değere göre 10 HU'dan az artış gösteren alanların nekroz olduğu kabul edildi. En geniş kesimlerinin göründüğü kesitte lezyonların en az 2/3'ünü içine alacak büyüklükte region of interest (ROI)'ler çizildi. Her ROI için dansite değeri (HU) kaydedildi. Bu dansite değerleri kullanılarak mutlak (MKYY) ve bağıl (BKYY) kontrast yıkanma yüzdeleri hesapland.. MKYY ve BKYY için su formüller kullanıldı:

- $\quad$ MKYY $=([$ portal faz ortalama dansite değeri-15.dakika ortalama dansite değeri] / [portal faz ortalama dansite değeri-prekontrast ortalama dansite değeri]) x 100

- $\quad$ BKYY $=([$ portal faz ortalama dansite değeri-15.dakika ortalama dansite değeri] / portal faz ortalama dansite değeri) x 100 (9).

\section{Bulgular}

Toplam 15 hasta çalışmaya dahil edildi. Hastaların 8'i kadın, 7'si erkekti. Ortanca (aralık) yaş 53 yıl (31-74 yıl) olarak hesaplandı. 15 lezyonun 9'u (\%60) hormonal olarak fonksiyonel, 6's1 (\%40) nonfonksiyoneldi. Fonksiyonel lezyonların 5'i androjen, 4'ü kortizon üretmekteydi. Hasta bilgileri Tablo 1'de özetlenmiştir.

Tablo 1. Hastaların demografik özellikleri, lezyonların patolojik tanı yöntemleri ve fonksiyonel durumları

\begin{tabular}{ll}
\hline Özellik & Değer \\
\hline Hasta & 15 \\
Cinsiyet & \\
\multicolumn{1}{l}{ Kadın } & $8(\% 53)$ \\
$\quad$ Erkek & $7(\% 47)$ \\
Yas \{yl\}, ortanca (aralık) & $53(31-74)$ \\
Tanı yöntemi & \\
$\quad$ Cerrahi & $12(\% 80)$ \\
$\quad$ Cerrahi eşliğinde biyopsi & $3(\% 20)$ \\
Fonksiyonel durum & \\
$\quad$ Fonksiyonel & $9(\% 60)$ \\
$\quad$ Non-fonksiyonel & $6(\% 40)$ \\
\hline
\end{tabular}

Lezyonların patolojik tanısı cerrahi $(\mathrm{n}=12)$ veya cerrahi eşliğinde biyopsi $(\mathrm{n}=3)$ ile konuldu. Kalitatif BT bulguları değerlendirildiğinde 13 lezyonda (\%93) nekroz varken sadece 1 lezyonda (\%7) nekroz yoktu. Bir hastada prekontrast faz elde olunmadığ 1 için nekroz varlığı araştırılamadı. Lezyonlardan 1'inde makroskobik yağ, 3'ünde milimetrik kalsifikasyon görüldü. Şekil 1'de makroskobik yağ ve Şekil 2'de milimetrik kalsifikasyonlar içeren AKK'ler görülmektedir. Tüm lezyonlar iyi sınırlı olup heterojen kontrastlanma göstermekteydi.

Kantitatif değerlendirmede lezyonların ortanca (aralık) boyutu 9,7 cm (6,3-18 cm) idi. Hastaların 14' ünde prekontrast faz, 8'inde arteriyel faz, 13' ünde portal faz ve 9'unda 15. dakika geç faz elde olunmuştu. $\mathrm{Bu}$ fazlarda ortanca (aralık) dansite değerleri sırasıyla şöyleydi: 34 HU (22-41 HU), 46 HU (27-65 HU), 60 HU (29-90 HU) ve 48 HU (28-64 HU) (Şekil 3). 15. dakika geç faz alınmış hastaların ortanca (aralık) mutlak ve bağıl kontrast yıkanma yüzdeleri (MKYY ve BKYY) sirasiyla \%48,8 (\%-3,8-62,5) ve \%21 (\%-1,5$30,8)$ idi. Şekil 4'te MKYY ve BKYY değerleri sirasıly \%52 ve $\% 30$ olan bir AKK'nin prekontrast, portal faz ve 15. dakika geç faz 
BT görüntüleri görülmektedir. Sadece bir lezyonun MKYY değeri $\% 60$ 'nn üzerindeydi $(\% 62,5)$. Bu lezyonun BKYY değeri \%15,1 olarak hesapland1. Lezyon bilgileri Tablo 2'de özetlenmiştir.

Şekil 1. Aksiyel prekontrast BT kesitinde sol adrenal bez yerleşimli 12,8x12,7 cm boyutlarında, global dansitesi 41 HU ölçülen, iyi sınırlı kitle ve kitle içerisinde $5 \mathrm{~mm}$ çapında ortalama dansitesi -38 HU ölçülen makroskobik yağ odağı görülmekte. Yapılan cerrahi sonrası lezyonun adrenal kortikal karsinom olduğu patolojik olarak doğrulandı.

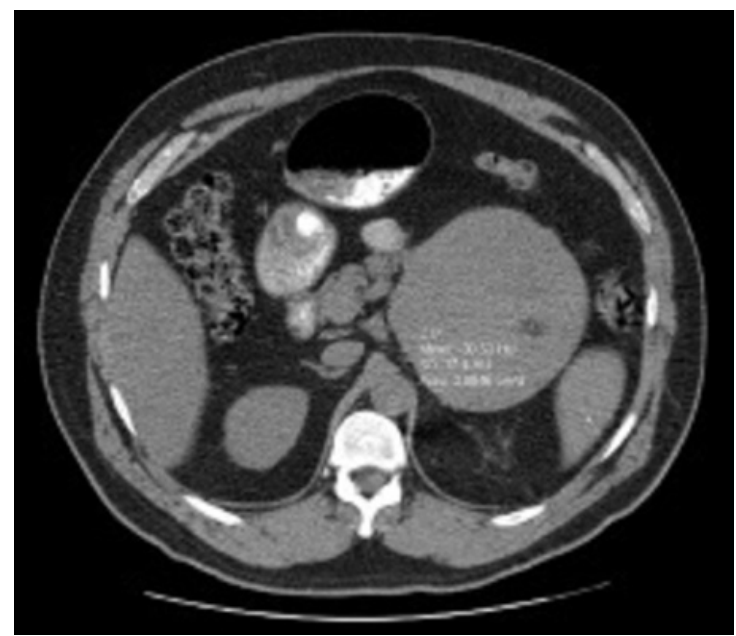

Şekil 2. Aksiyel prekontrast BT kesitinde sağ adrenal bez yerleşimli 7,4x6,7 cm boyutlarında, ortalama dansitesi $33 \mathrm{HU}$ ölçülen, iyi sınırlı kitle ve kitle içerisinde noktasal kalsifikasyon odakları görülmekte. Adrenal kortikal karsinom tanısı cerrahi ile doğrulandı.

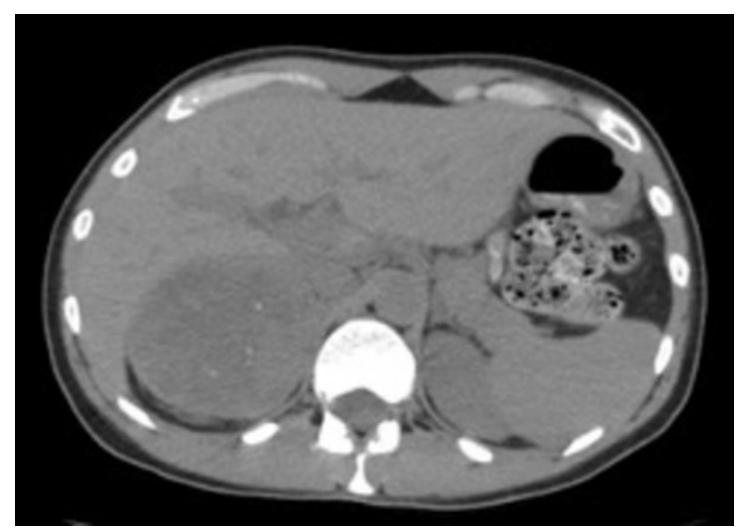

Şekil 3. Lezyonların prekontrast, arteriyel faz, portal faz ve 15. dakika geç fazlardaki dansite değerlerini gösteren diagram. Her renk bir hastayı temsil etmektedir.

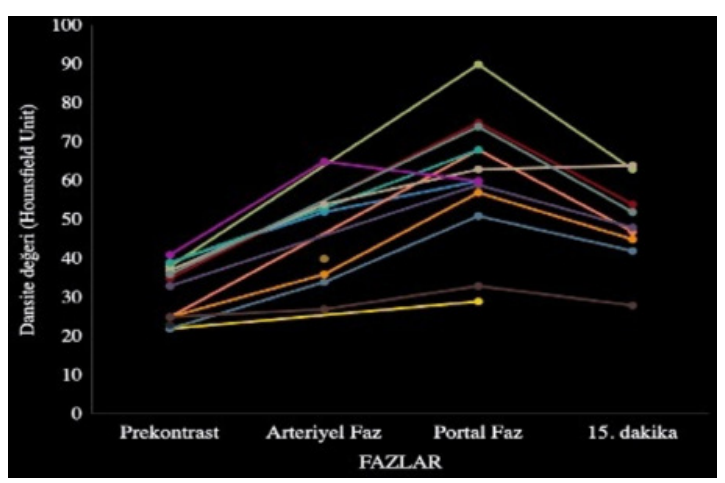

Adrenal Kortikal Karsinom'da BT Bulguları - Altınmakas
Şekil 4. Aksiyel prekontrast (A), portal faz (B) ve 15. dakika geç faz (C) BT kesitlerinde sağ adrenal bez yerleşimli 7,2x6,6 cm boyutlarında, iyi sınırl, kalsifikasyon veya yağ barındırmayan, heterojen iç yapıda kitle görülmekte. Kitlenin ortalama dansitesi sırasıyla 38 HU, 90 HU ve 63 HU ölçülmüş olup buna göre mutlak ve bağıl kontrast yıkanma yüzdeleri $\% 52$ ve $\% 30$ olarak hesaplandı. Oranlar “adenom-dışı patoloji' tanısını desteklemektedir. Yapılan cerrahi ile adrenal kortikal karsinom tanısı doğrulandı.

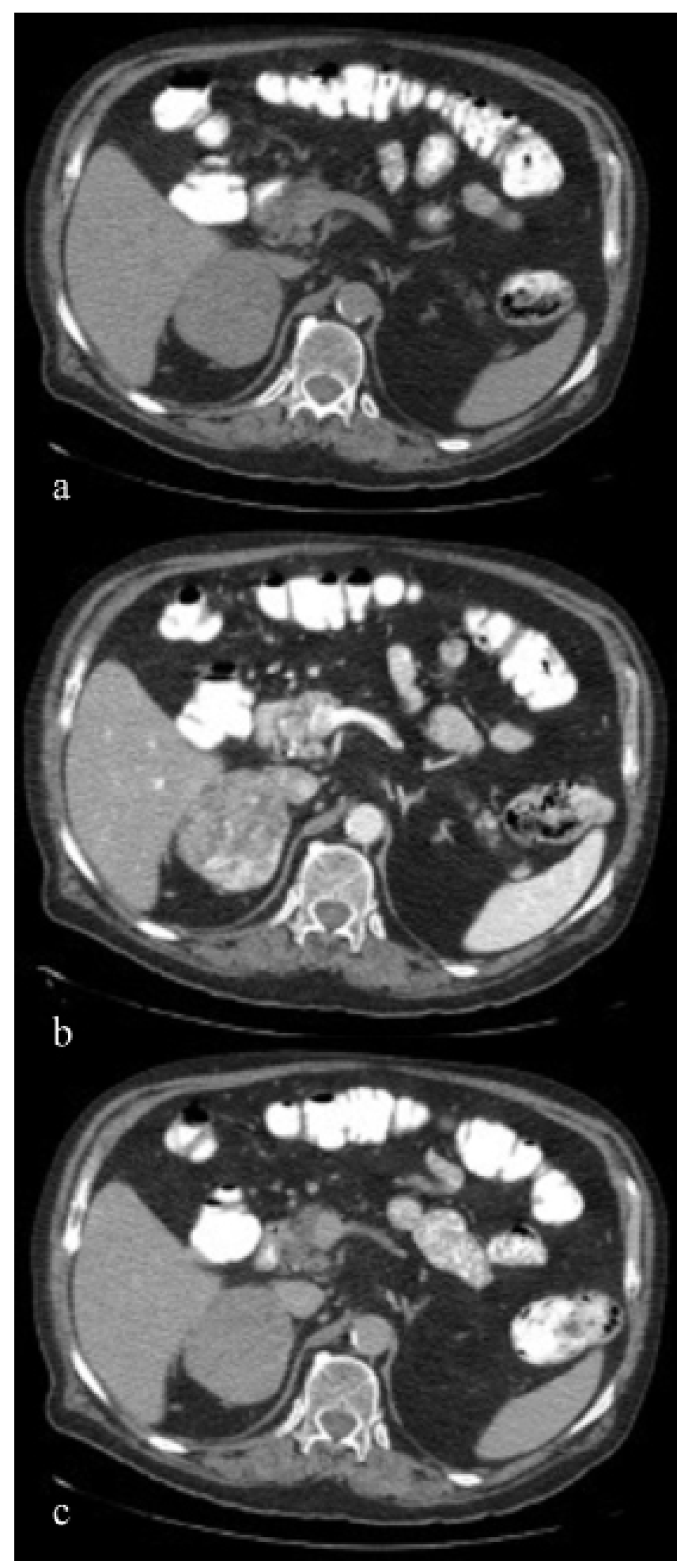

Genel Tip Derg 2021;31(3)299-303 
Tablo 2. Lezyonların kalitatif ve kantitatif BT bulguları

\begin{tabular}{|c|c|}
\hline Özellik & Değer \\
\hline Lezyon & 15 \\
\hline \multicolumn{2}{|l|}{ Kantitatif Özellikler } \\
\hline Boyut - uzun aks $\{\mathrm{cm}\}$, ortanca (aralık) & $9,7 \mathrm{~cm}(6,3-18 \mathrm{~cm})$ \\
\hline \multicolumn{2}{|l|}{ Ortanca (aralık) dansite değeri (HU) } \\
\hline Prekontrast $(\mathrm{n}=14)$ & $34 \mathrm{HU}(22-41 \mathrm{HU})$ \\
\hline Arteriyel faz $(n=8)$ & $46 \mathrm{HU}(27-65 \mathrm{HU})$ \\
\hline Portal faz $(n=13)$ & $60 \mathrm{HU}(29-90 \mathrm{HU})$ \\
\hline 15. dakika geç faz $(n=9)$ & $48 \mathrm{HU}(28-64 \mathrm{HU})$ \\
\hline \multicolumn{2}{|l|}{ Ortanca (aralık) kontrast yıkanması (\%) } \\
\hline Mutlak & $\% 48,8(\%-3,8-62,5)$ \\
\hline Bağıl & $\% 21(\%-1,5-30,8)$ \\
\hline \multicolumn{2}{|l|}{ Kalitatif Özellikler } \\
\hline Taraf (sağ/sol) & $8 / 7$ \\
\hline Kalsifikasyon (var/yok) & $3 / 12$ \\
\hline Makroskobik yağ (var/yok) & $1 / 14$ \\
\hline Nekroz (var/yok) & $13 / 1$ \\
\hline \multicolumn{2}{|l|}{ Sinır } \\
\hline İyi sınırlı & $15(\% 100)$ \\
\hline Silik sınırı & $0(\% 100)$ \\
\hline \multicolumn{2}{|l|}{ Kontrastlanma paterni } \\
\hline Homojen & $0(\% 0)$ \\
\hline Heterojen & $15(\% 100)$ \\
\hline
\end{tabular}

\section{Tartışma}

AKK'ler adrenal korteksten kaynaklanan malign tümörler olup, tüm adrenal insidentalomaların \%5'inden azını oluşturmaktadır (12). Erken çocukluk dönemi ( $<5$ yaş) ve orta yaş (4-5. dekad) olmak üzere iki farklı yas grubunda sıklığının arttığı bilinmektedir (12). Yaklaşık \%60'1 hormonal olarak aktif olup kortizon, aldosteron ve seks hormonları salg1layabilirler (12).

AKK'ler büyük tümörler olup \%70 kadarı tanı anında 6 cm'nin üzerindedir (5). Tipik olarak makroskobik yağ içermedikleri bilinmekte birlikte literatürde makroskobik yağ içeren AKK'ler de bildirilmiştir $(13,14)$. Fischman ve ark. yaptığı çalışmada 38 AKK'nin 9'unda (\%24) kalsifikasyon saptandığı raporlanmış (15). Bizim çalışmamızda literatürdekine benzer oranlar saptadık; 15 lezyonun 9'u (\%60) hormonal olarak aktifti, tüm lezyonlar 6 cm'den daha büyüktü, sadece 1 lezyonda (\%7) makroskobik yağ, 3 lezyonda $(\% 20)$ da milimetrik kalsifikasyon vardı.

AKK'lerin prekontrast ortalama dansite değerlerinin 20 HU'nun üzerinde olduğu bilinmektedir $(10,11,16)$. Petersen ve ark. 51 AKK üzerinde yaptığ 1 çalışmada prekontrast ortanca (aralık) dansite değeri $34 \mathrm{HU}$ (14-74 HU) bulunmuş. AKK tanısı için >21 HU tanı kriteri olarak kabul edildiğinde duyarlılık ve özgüllük sırasıyla \%96 ve \%80 olarak bildirilmiş (16). Bir diğer çalışmada Szolar ve ark. 11 AKK ile 56 AKK-dışı lezyonun BT bulgularını karşılaştırmış ve AKK'lerin ortalama (aralık) prekontrast dansite değerini 39 HU (23$52 \mathrm{HU}$ ) olarak rapor etmişlerdir (10). Slattery ve ark. 7 AKK'nin dahil edildiği çalışmada lezyonların ortalama (aralık) prekontrast dansite değerini 39 HU (32-45 HU) olarak bulmuşlardır (11). Kontrast sonras1 görüntülemede ise AKK'lerin genellikle heterojen kontrastlandıkları ve nekroz içerdikleri bildirilmiştir (17). Bizim çalışmamızda da yukarıda bahsedilen çalışmalardaki gibi tüm lezyonların prekontrast dansite değerleri 21 HU'nun üzerinde bulundu. Ortanca (aralık) prekontrast dansite değeri $34 \mathrm{HU}$ (22-41 HU) idi. Tüm lezyonların kontrastlanması heterojen olup 1 lezyon hariç tamamında nekroz görüldü. Arteriyel faz görüntüleme adrenal BT protokollerinde yer almamaktadır. Mevcut çalışmamızda 8 hastaya ait arteriyel faz görüntüler mevcut olup dansite değerleri 27-65 HU aralığında idi. Bu 8 hastada arteriyel faz daniste değerlerinin portal fazdakinden düşük olduğunu gözlemledik.

Literatürde adrenal kitlelerin BT ile değerlendirilmesinde, adenomları adenom dışı lezyonlardan ayrıt etmek için kullanılan kontrast yıkanma yüzdesi kriterlerinin (MKYY $>\% 60$ ve BKYY> \%40) AKK'lerdeki etkinliğini araştıran çok az sayıda çalışma bulunmaktadır $(10,11)$. Szolar ve ark. yaptığı çalışmaya 11 AKK dahil edilmiş olup geç faz görüntüler yaygın bir şekilde kullanılan 15. dakika yerine kontrast sonrası 10. dakika elde olunmuştur. Sonuç olarak AKK'lerin adenomlara göre daha yavaş kontrast y1kanması gösterdiği bildirilmiştir (10). Diğer bir çalışmada Slattery ve ark 7 AKK'nin KYY'lerini değerlendirmiş ve Szolar ve ark ile benzer şekilde AKK'lerin adenom benzeri kontrast yıkanması göstermediği kaydedilmiştir (11). Bu çalışmada ise geç faz görüntüler 6 hastada 10 dakika veya daha önce elde edilmiştir.

Bizim çalışmamız AKK'lerin 15. dakika geç faz kontrast yıkanma değerlerini gösteren literatürde ilk çalışmadır. Çalışmamıza dahil 9 hastanın 15. dakika geç faz imajları mevcuttu ve ortanca (aralık) MKYY ve BKYY değerleri sirasiyla \%48,8 (\%-3,8-62,5) ve $\% 21$ $(\%-1,5-30,8)$ olarak hesaplandı. Tüm lezyonların BKYY değeri adenom-dışı patolojilerde beklendiği gibi \%40'ın altındaydı. Benzer şekilde 9 lezyondan 8'inin MKYY değeri adenom-dışı patolojiler ile uyumluydu $(<60 \%)$. Sadece 1 lezyonun MKYY değeri \%60'tan büyük olup $(\% 62,5)$ lezyonun neredeyse tümüyle nekrotik olması ile ilgili olabileceği değerlendirildi.

Çalışmamızın temel sınırlılı̆̆ Bir diğer sınırlılık, vaka sayımızın çok geniş olmamasıdır ki bu da AKK'nin nadir görülen bir tümör olmasından kaynaklanmaktadır.

\section{Sonuç}

AKK'ler BT'de genellikle büyük $(>6 \mathrm{~cm})$, iyi sınırlı, tek tarafll, heterojen iç yapıda ve nekrotik kitleler olarak görülürler. Sıklıkla olmasa da makroskobik yağ ve kalsifikasyon içerebilirler. Prekontrast dansiteleri 20 HU'dan yüksek olup geç faz imajlarda adenomlara göre daha az kontrast yıkanması gösterirler. Radyologların bu nadir görülen tümörlerin tipik görüntüleme özelliklerini aşina olmaları tanıyı kolaylaştıracaktır.

\section{Kaynaklar}

1.Reznek RH, Narayanan P. Primary adrenal malignancy. Husband \& Reznek's imaging in oncology, 3rd ed. London, UK: Informa Healthcare-2010;280-298

2.Latronico, Ana C., and George P. Chrousos. "Adrenocortical tumors." The Journal of Clinical Endocrinology \& Metabolism 82.5-1997; 1317-1324.

3.Wooten MD, King DK. Adrenal cortical carcinoma: epidemiology and treatment with mitotane and a review of the literature. Cancer-1993; 72: 3145-3155

4.Ng L, Libertino JM. Adrenocortical carcinoma: diagnosis, evaluation and treatment. J Urol-2003; 169:5-11

Adrenal Kortikal Karsinom'da BT Bulguları - Altınmakas 
5.Bharwani, N., Rockall, A. G., Sahdev, A. et al. Adrenocortical carcinoma: the range of appearances on CT and MRI. American journal of roentgenology-2011;196(6), W706-W714.

6.Altinmakas, E., Guo, M., Kundu, U. R., Habra MA, Ng CS . Computed tomography and $18 \mathrm{~F}$-fluorodeoxyglucose positron emission tomography/computed tomography findings in adrenal candidiasis and histoplasmosis: two cases. Clinical imaging-2015;39(6), 11151118 .

7.Altinmakas, E., Üçısık-Keser, F. E., Medeiros, Ng CS CT and 18F-FDG-PET-CT Findings in Secondary Adrenal Lymphoma with Pathologic Correlation. Academic radiology-2019;26(6), e108-e114.

8.Altinmakas, E., Perrier, N. D., Grubbs, E. G., Lee EJ, Prieto VG, Ng CS Diagnostic performance of adrenal $\mathrm{CT}$ in the differentiation of adenoma and pheochromocytoma. Acta Radiologica-2020;61(8), 1080-1086.

9.Korobkin M, Brodeur FJ, Francis IR, Quint LE, Dunnick NR, Goodsitt M Delayed enhanced CT for differentiation of benign from malignant adrenal masses. Radiology-1996; 200:737-742

10.Szolar, D. H., Korobkin, M., Reittner, P. et al. Adrenocortical carcinomas and adrena pheochromocytomas: mass and enhancement loss evaluation at delayed contrastenhanced CT. Radiology-2005; 234(2), 479-485.

11.Petersenn, S., Richter, P. A., Broemel, T. et al. Computed tomography criteria for discrimination of adrenal adenomas and adrenocortical carcinomas: analysis of the German ACC registry. Eur J Endocrinol-2015; 172(4), 415-22.

12.Allolio B, Fassnacht M. Clinical review: adrenocortical carcinoma - clinical update. J Clin Endocrinol Metab-2006; 91:2027-2037

13.Egbert, N., Elsayes, K. M., Azar, S., Caouli M. Computed tomography of adrenocortical carcinoma containing macroscopic fat. Cancer Imaging,-2010;10(1), 198.

14.Heye, S., Woestenborghs, H., Van Kerkhove, F., Oyen R Adrenocortical carcinoma with fat inclusion: case report. Abdominal imaging-2005;30(5), 641-643.

15.Fishman, E. K., Deutch, B. M., Hartman, D. S., Goldman SM, Zerhouni EA, Siegelman SS. Primary adrenocortical carcinoma: CT evaluation with clinical correlation. American Journal of Roentgenology-1987; 148(3), 531-535.

16.Slattery, J. M., Blake, M. A., Kalra, M. K. et al. Adrenocortical carcinoma: contrast washout characteristics on CT. American Journal of Roentgenology-2006; 187(1), W21-W24.

17.Shin, Y. R., \& Kim, K. A. Imaging features of various adrenal neoplastic lesions on radiologic and nuclear medicine imaging. American Journal of Roentgenology-2015; 205(3), 554-563. 